\title{
Advanced Prostate Adenocarcinoma
}

National Cancer Institute

\section{Source}

National Cancer Institute. Advanced Prostate Adenocarcinoma. NCI Thesaurus. Code C156286.

A prostatic adenocarcinoma that has spread extensively to other anatomical sites or is no longer responding to treatment. 